\title{
Distributed Control of Battery Energy Storage Systems for Improved Frequency Regulation
}

DOI:

10.1109/TPWRS.2020.2974026

\section{Document Version}

Accepted author manuscript

Link to publication record in Manchester Research Explorer

\section{Citation for published version (APA):}

Zhao, T., Parisio, A., \& Milanovic, J. V. (2020). Distributed Control of Battery Energy Storage Systems for Improved Frequency Regulation. I E E E Transactions on Power Systems.

https://doi.org/10.1109/TPWRS.2020.2974026

\section{Published in:}

I E E E Transactions on Power Systems

\section{Citing this paper}

Please note that where the full-text provided on Manchester Research Explorer is the Author Accepted Manuscript or Proof version this may differ from the final Published version. If citing, it is advised that you check and use the publisher's definitive version.

\section{General rights}

Copyright and moral rights for the publications made accessible in the Research Explorer are retained by the authors and/or other copyright owners and it is a condition of accessing publications that users recognise and abide by the legal requirements associated with these rights.

\section{Takedown policy}

If you believe that this document breaches copyright please refer to the University of Manchester's Takedown Procedures [http://man.ac.uk/04Y6Bo] or contact uml.scholarlycommunications@manchester.ac.uk providing relevant details, so we can investigate your claim.

\section{OPEN ACCESS}




\title{
Distributed Control of Battery Energy Storage Systems for Improved Frequency Regulation
}

\author{
Tianqiao Zhao, Alessandra Parisio, Senior Member, IEEE, Jovica V. Milanović, Fellow, IEEE,
}

\begin{abstract}
In this paper a distributed control strategy for coordinating multiple battery energy storage systems to support frequency regulation in power systems with high penetration of renewable generation is proposed. The approach is based on an online convex optimisation framework that considers both the operating costs of storage systems and the frequency regulation requirements. The proposed framework uses a dynamic regret based on the last available information up to the current point in time of renewable generation and demand, which are uncertainty sources. The approach provides more accurate control than the currently employed prediction-based algorithms and it can be implemented in a distributed manner using a multi-agent system framework that facilitates plug-and-play functionality. The effectiveness and scalability of the proposed strategy is assessed through several case studies.
\end{abstract}

Index Terms-Distributed optimisation, frequency response, online convex optimisation, battery energy storage systems, frequency-related constraints, uncertain renewable generation

\section{INTRODUCTION}

The increasing penetration of renewable generation in power systems resulted in a considerable part of conventional synchronous generators (SGs) providing system inertial and primary frequency response (PFR) being replaced by renewable energy sources (RES). However, these RES contribute less, if at all, inertia and frequency regulation to a power system [1]. As a result, the total system inertia is reduced as well as the frequency control ability of the system, which poses new challenges to the frequency stability of power grids [2].

To mitigate the challenges caused by the high share of RES, new technical solutions are explored by several system operators worldwide [3], in particular faster-acting frequency response services. National Grid (NG), the Great Britain transmission system operator, has introduced an ancillary service named Enhanced Frequency Response (EFR) to provide a fast and dynamic frequency regulation service [4] and improve the management of system frequency pre-fault to maintain system frequency closer to $50 \mathrm{~Hz}$. It is a dynamic service with high revenue requiring the asset providing it to change its power output proportional to any frequency deviation in real time on a second-by-second basis. Few assets are suitable for providing this dynamic service. The emerging fast-response battery energy storage systems (BESS) provide a promising solution to provide dynamic fast frequency response and improve the frequency response of the reduced inertial power system, as they are able to compensate system power imbalance instantly [5], [6]. Extensive efforts [7]-[9] have been made to take advantage of the ability of fast-response BESS to improve frequency response and some practical applications [10]-[12] have demonstrated their feasibility and flexibility to do so.

The majority of the existing methods, however, are centralized and they would require a central controller to collect, process and distribute the information from/to all the computing units and local controllers of the various BESS. Therefore, they are not feasible to coordinate a large number of battery units in real time to provide dynamic fast frequency response due to high computational and communication requirements and a high sensitivity to noise, communication delays and single point failures [13].

Distributed algorithms relying on local and neighbouring information, on the other hand, can not only reduce computation time, but also account for system topology changes by taking advantage of local intelligent controllers [14], which are able to communicate with other neighboring controllers and execute simpler algorithms locally. The recent applications of distributed algorithms in power systems [15]-[17] include, among the other, real-time control of BESS to solve the security-constrained DC optimal power flow [16] and to BESS coordinated control to maintain the power balance at minimum cost [17]. All of them rely on accurate predictions of renewable generation output and/or load demand, which is very difficult to accomplish considering their inherent variability, in order to be effective. Due to the high penetration of renewable generation, its unpredictable parts would have increasing affects on the system stability. As a result, these uncertain and unpredictable characteristics should be taken into account when designing a more accurate control strategy for BESS.

The online convex optimisation (OCO) [18] has recently gained attention in power system applications [19]-[21], particularly in demand-side management applications, since it is computationally efficient and suitable for real-time implementation. In fact, OCO requires less information compared with algorithms using both real-time and forecast information and it is an optimisation method that learns from experience as more aspects of the problem are observed.

Statement of contribution: In this study two main research questions are addressed: $i$ ) how to optimise and coordinate a large and arbitrary number of BESS for the frequency control in real-time; $i$ i) how to take uncertainties in both network and BESS operation into the control framework while accounting for BESS degradation and revenue to BESS owners. Traditional frequency control methods (e.g., droop-based control) are not suitable for addressing the above challenges and novel control approaches are required.

The focus is on the dynamic fast frequency response provision to compensate for frequency drops, which is what is currently required. This entails that the devised control algorithm optimises and coordinates the BESS discharging behaviour. We remark that the proposed framework can be easily adapted to optimise the charging behaviour if the BESS are required to contain the frequency rises.

A methodology combining consensus algorithms and online convex optimisation is proposed for cost-effective and coordinated control of distributed BESS to provide a frequency 
regulation service in power systems with RES. Existing algorithms only consider one of the aforementioned methods individually, i.e., consensus algorithms [14], [17] and online convex optimisation [19]-[21]. The proposed control framework combines advantages of both algorithms, in particular the little information requirement and the fully distributed and computationally beneficial implementation.

A time-varying optimisation problem to maximise BESS welfare is first formulated, which maximises the reward price and minimises the operating costs of each battery device, while satisfying frequency-related constraints to improve system frequency response. An OCO and consensus-based distributed algorithm is proposed to restore system frequency to the nominal value optimally and timely by controlling the power output from BESS. Using a multi-agent system framework, each battery device is regarded as an agent. A distributed consensus-based algorithm is adopted by each agent to estimate the system information (e.g., system active power supply-demand mismatch) locally. Then each agent exchanges information only with its neighbouring agents through a local communication network and therefore, the communication and computational burden is reduced. The proposed approach is less sensitive to the single-point failure and enables a plug-andplay functionality even during a frequency drop. Furthermore, the proposed algorithm solves the time-varying optimisation problem without prior knowledge about renewable generation and load demand. The effectiveness and scalability of the distributed approach are illustrated on case studies considering the IEEE 14-bus and 33-bus test systems modified to incorporating RES. The results demonstrate the suitability of the proposed algorithm for implementation in a large-scale power system with RES. The simulation study was performed on a laptop with $2.4 \mathrm{GHz}$ Intel Core I5 CPU: i5 2557m @ 1.7 $\mathrm{GHz}$, and the problem was formulated, coded and solved in the MATLAB 2016b environment.

\section{BACKGROUND}

In this section the OCO framework is outlined and Table I defines parameters and variables used in this paper.

\section{A. Online Convex Optimisation}

In the OCO framework, a player plays a repeated game in a time horizon $T$ [18]. Denoting $t \in \mathcal{T}$ as the per time slot and $\mathcal{T}$ be the time slot set, a player selects an action $x_{t}$ from a convex set $\mathcal{X} \in \mathbb{R}^{n}$ and suffers a loss $f_{t}\left(x_{t}\right)$, where $f_{t}(\cdot): \mathbb{R}^{n} \rightarrow \mathbb{R}$ is the loss function. To account for uncertain and time-varying renewable generation and load demand, the traditional OCO setting is broadened from a fixed environment to an unknown and time-varying environment. In this modified optimisation problem, the player is the a battery with a timevarying objective function in terms of the operating cost and the frequency regulation reward. The decision to be made is how to control the outputs of BESS to compensate the active power imbalance cost-effectively. In order to account for the uncertainties associated with RES and demand, a time-varying environment is considered, i.e., there are timevarying penalty functions $g_{t}(\cdot): \mathbb{R}^{l} \rightarrow \mathbb{R}$ and $h_{t}(\cdot): \mathbb{R}^{m} \rightarrow \mathbb{R}$, which leads to time-varying constraints. To make the OCO scheme compatible with the considered dynamic and uncertain environment, an improved performance index, a dynamic regret, which compares the performance of the OCO scheme to the sequence of optimal solutions, is applied [22], i.e., $R_{T}^{d}:=\sum_{t \in \mathcal{T}} f_{t}\left(x_{t}\right)-\sum_{t \in \mathcal{T}} f_{t}\left(x_{t}^{*}\right)$, where $x_{t}^{*}$ is the sequence of best dynamic decisions given as $x_{t}^{*} \in \arg \max _{x \in \mathcal{X}} f_{t}(x)$, s.t. $g_{t}(x) \leq 0, h_{t}(x)=0, \forall t \in[0, T]$. In this case, the goal of an OCO algorithm is to generate decisions with a sub-linear regret as a function of $T$ [22], where $R_{T}^{d}=\mathcal{O}(\sqrt{T})$ and consequently, $\lim _{T \rightarrow \infty} \frac{R_{T}^{d}}{T}=0$. This effectively means that the online algorithm asymptotically converges to the sequence of best dynamic schedules.

\section{B. Model of the communication network}

In the test network, $N_{B}$ distributed BESS are included as controllable agents. The communication among BESS is governed by a strong connected and weighted digraph $\mathcal{G}=(\mathcal{V}, \mathcal{E}, \mathcal{W})$, where $\mathcal{V}=\left\{\nu_{1}, \ldots, \nu_{N_{B}}\right\}$ denotes the agent set and $\mathcal{E}$ is the edge set. There exists a direct path $(i, j) \in \mathcal{E} \subseteq \mathcal{V} \times \mathcal{V}$ if and only if the $i$ th battery agent can receive information from the $j$ th battery agent. We assume that the weight matrix of $\mathcal{G}$, i.e., $\mathcal{W} \in \mathbb{R}^{N_{B} \times N_{B}}$, is doubly stochastic, and the weight of an edge from the $i$ th agent to the $j$ th agent is $w_{i j}>0$ if and only if $(i, j) \in \mathcal{E}$ and $w_{i j}=0$ otherwise. In this network, each agent receives information from its in-neighbours $\mathcal{N}_{i}^{+}$and sends information to its out-neighbours $\mathcal{N}_{i}^{-}$, and the in-degree and out-degree of the $i$ th agent are defined by $d_{i}^{+}=\left|\mathcal{N}_{i}^{+}\right|$and $d_{i}^{-}=\left|\mathcal{N}_{i}^{-}\right|$ respectively, where $|\cdot|$ is the cardinality of a set. As indicated in [23], the designed communication network is independent of the power network, and hence it can be constructed in a cost-effective way based on practical requirements. While the communication network can be theoretically assumed to be independent of the power network, this is not necessarily true in practice as many power lines are used as information carries, i.e., the topology is very similar at basic level.

Remark 2.1: Note that the communication delay is a nonnegligible factor when implementing a distributed algorithm. Efficient approaches have been proposed to handle the communication delays through optimising the communication network, augmenting the communication graph and design a learning parameter for the largest delay [24], [25]. Thus, the effects of the communication delay on the distributed algorithms would be reduced or even eliminated when the communication delay is bounded. Analysing into detail the impact of the communication network design on the proposed control framework and exploring the most suitable design approach for the communication network were note within the scope of this study and will be addressed in the future.

\section{BESS OPTIMAL MANAGEMENT}

As remarked in Section I, the overall system inertia is reducing due to the increasing penetration of RES and the dynamics of the system frequency response change. SGs may not be able to respond to power imbalances immediately due to the ramping rate limit and large inertia, which could be assumed to be unchanged for a short-term frequency excursion [7], [16]. Therefore, to ensure an appropriate frequency response and 
TABLE I

VARIABLES AND PARAMETERS

\begin{tabular}{ll}
\hline$P_{B, t}^{i}$ & nonnegative power output of $i$ th battery unit \\
$\Delta P_{M}(t)$ & at time $t$ \\
$S o C_{B, t}^{i}$ & total active power mismatch at time $t$ \\
$f(t)$ & State of Charge of $i$ th battery unit at time $t$ \\
$\rho_{c}$ & system frequency at time $t$ \\
$R$ & marginal cost of degradation \\
$L_{N}$ & replacement cost of a battery cell $(\$ / \mathrm{Wh})$ \\
$p(t)$ & total number of operational cycles \\
$\eta^{i}$ & reward price at time $t$ \\
$\xi_{i, t}$ & discharging efficiency of $i$ th battery unit \\
$C a^{i}$ & inner energy rate (IER) of $i$ th battery unit at \\
$P_{B}^{i, \min } / P_{B}^{, i m a x}$ & time t \\
& ith BESS capacity \\
$E_{\max } / E_{\min }$ & minimum/maximum active power constraint \\
$S o C_{B}^{i, \min } / S o C_{B}^{i, \text { max }}$ & of $i$ th battery unit \\
& minimum/maximum energy level of a battery \\
$\kappa, \epsilon$ & positive stepsizes \\
$\Delta t_{c}$ & time-slot duration \\
$\mathcal{B}$ & set of BESS constraints \\
$\mathcal{T}$ & set of service time horizon \\
$\mathcal{F}$ & feasible set of the optimisation problem \\
\hline &
\end{tabular}

ultimately the frequency stability, fast controllable devices, such as power electronic-based BESS, have been installed in modern power grids to provide frequency support. The response time of BESS could be neglected since they have fast electronic control and they are able to ramp up to full capacity within ten to one hundred milliseconds [11], [26].

To facilitate a cost-effective frequency support, a battery storage system should meet the following requirements: $i$ ) to deliver/absorb power quickly in response to observed frequency deviations; ii) to be operated considering specific constraints, including power output limits, State-of-Charge (SoC) limits, to avoid over-charging/discharging; iii) to have a response designed considering the operating costs (inner energy rate and degradation cost). The optimisation problem therefore needs to be formulated to take into account all the above requirements.

\section{A. Cost Function}

To control BESS economically, the following operational costs should be considered in their management.

1) Inner energy rate: when using a battery system for frequency regulation, the power loss is a non-negligible factor that would have effects on its charging/discharging behaviour and output response. In order to account for the loss factor, a coefficient $\xi_{i}$, i.e., $\xi_{i}=\Delta E_{B, i} / P_{B}^{i}$, has been introduced, where $\Delta E_{B, i}$ is the inner consumption energy of battery $i$ at $P_{B}^{i}$ [27]. Experiments in [27] have shown that IER can be represented by a piecewise linear function by applying an approximation method to linearise the IER function within a given SoC range. However, in the practical application of frequency control, the characteristics of IER could be different from the estimated/linearised ones under different operational conditions. Thus, a time-varying function is adopted in this study to indicate the IER characteristics, i.e.,

$$
\xi_{i, t}:=a_{i, t} P_{B, t}^{i}+b_{i, t},
$$

where $a_{i, t}$ and $b_{i, t}$ are coefficients estimated based on the SoC and output power at $t$ [27]. The maximisation of the efficiency is achieved by minimising the following function

$$
C_{i, t}^{\mathrm{IER}}:=\xi_{i, t} P_{B, t}^{i}:=a_{i, t}\left(P_{B, t}^{i}\right)^{2}+b_{i, t} P_{B, t}^{i} .
$$

2) Degradation cost: the degradation cost of battery cells during repeated charging/discharging cycles plays an important role in the BESS operation. Different methods have been proposed in the past to investigate battery degradation behaviour [28], [29]. In this work a linearised battery degradation model is adopted, which has been widely used in the past in the optimal power control of BESS [28], [29]. It was shown in [29] that, assuming a limited operational region, the marginal cost of cycle ageing can be approximated by a constant value. In order to formulate $\rho_{c}$ the battery cycle life is normalized into amounts of energy drawn from/to the battery per charging/discharging cycle, assuming the battery cell works before end-of-life (EOL). Then, the battery degradation cost is expressed as

$$
C_{i, t}^{\text {Degradation }}:=\rho_{c} \times P_{B, t}^{i} .
$$

where $\rho_{c}=R /\left(2 L_{N} \times\left(\frac{E_{\max }-E_{\min }}{E_{\text {rated }}}\right)\right) * \Delta t_{c}$. The term in the cost function accounting for BESS degradation can be reformulated in order to focus on different aspects, e.g., the actual number of performed cycles, as long as the degradation cost function is expressed as a convex function. This would lead to a different interpretation of the battery degradation but would not have any impact on the effectiveness of the proposed approach.

\section{B. Problem formulation}

The use of BESS to provide fast frequency regulation is drawing an increasing interest [28]. NG launched the EFR service to encourage service providers, in particular BESS, to participate in the fast frequency regulation market [4]. Within EFR, service providers will be rewarded when meeting specific technical criteria designed by NG, which are: $i$ ) the response has to take place within 1s of frequency deviations incurring; ii) the service providers have to be able to deliver a minimal response of $1 \mathrm{MW}$. The proposed control framework aims at controlling BESS in order to provide EFR while considering their operating costs and balancing a real-time supplydemand mismatch. The payment received from the system operator following EFR market information is calculated by $p(t) P_{B, t}^{i}$. However the particularly challenging EFR technical requirements are considered in this study, the proposed control framework can be easily adapted to handle different frequency services. Denoting $P_{B}(t)=\left[P_{B, t}^{1}, \ldots, P_{B, t}^{N_{B}}\right]^{T}$ for $i=$ $\left[1, \ldots, N_{B}\right]$, and $C_{i, t}\left(P_{B, t}^{i}\right)=C_{i, t}^{\text {Degradation }}+C_{i, t}^{\mathrm{IER}}-p(t) P_{B, t}^{i}$, the operational problem, $\forall i \in \mathcal{B}, \forall t \in \mathcal{T}$, is formulated as 


$$
\begin{gathered}
\min _{P_{B, t}} \quad \sum_{t=1}^{T} C_{t}\left(P_{B, t}\right)=\sum_{t=1}^{T} \sum_{i \in \mathcal{B}} C_{i, t}\left(P_{B, t}^{i}\right) \\
\text { s.t. } \quad P_{B}^{i, \min } \leq P_{B, t}^{i} \leq P_{B}^{i, \max } \\
S o C_{B, t}^{i, \min } \leq S o C_{B, t}^{i}=S o C_{B, t-1}^{i}-\eta^{i} \frac{P_{B, t}^{i}}{C a^{i}} \leq S o C_{B, t}^{i, \max } \\
\sum_{i \in B} P_{B, t}^{i}=\varrho \Delta P_{M, t} \\
P_{B}^{i, \max }\left(-\frac{1}{\alpha} \frac{f(t)-f(t-1)}{\Delta t}-\beta\right)<P_{B, t}^{i}-P_{B, t-1}^{i} \\
<P_{B}^{i, \max }\left(-\frac{1}{\alpha} \frac{f(t)-f(t-1)}{\Delta t}+\beta\right),
\end{gathered}
$$

where $0<\varrho \leq 1$ is a weighting factor to scale the current power mismatch to the contracted BESS power capacity. To avoid over-discharging and over-charging, equation (4c) limits the operation of the $i$ th battery between $S o C_{B}^{i, \text { min }}$ and $S o C_{B, i}^{i, \max }$. To regulate the frequency to the nominal value, the constraints (4d) - (4e) are formulated, where (4d) is formulated to maintain the system balance under abnormal conditions. As required by the EFR service, in order to avert stability problems caused by sudden and quick BESS responses, a ramp-rate limitation proportional to Rate of Change of Frequency (RoCoF) is to be included in the optimisation problem, i.e., $(4 \mathrm{e})$, where $f(t)$ and $f(t-1)$ are the measurements of the system frequency at $t$ and $t-1$, respectively. The ramp-rate can be tuned through the parameters $\alpha$ and $\beta$ according to performance requirements [30].

Remark 3.1: Since the introduced fast frequency services are mainly designed for compensating for frequency drops [31], this paper focuses on the discharging mode. However, the proposed control algorithm can be easily adopted to support the grid in response to frequency rise events by replacing the discharging efficiency in (4c) with the charging efficiency and considering a positive instead of a negative sign. Furthermore, time-varying limits on the BESS power and capacity can be easily integrated in the proposed framework.

Remark 3.2: A power system with wind turbines would have a modified swing equation as indicated by several studies in the literature [32], [33]. However, the proposed control framework is still fully applicable and its performance does not depend on the system frequency modelling. The system frequency modelling is adopted to facilitate the formulation of (4e) and so the change in the swing equation would only affect the selection of the tuning parameters $\alpha$ and $\beta$, which depends on changes in the system frequency [34]. Therefore, a power system with high penetration of renewable generation would have a different setting of $\alpha$ and $\beta$ [30].

Remark 3.3: Fast frequency response services commonly require the assets delivering the service for a minimum period of time (e.g., 15 minutes for EFR [4]). Delivery beyond this time period is not a requirement and the assets will not be penalised for ceasing the service delivery if needed. A deadband is designed to enable BESS' SoC recovery $( \pm 0.05 \mathrm{~Hz}$ or \pm 0.015 for EFR [4]). Within the specified deadband the battery assets can manage their state of charge so as to ensure they are prepared to provide a continuous service in the future. BESS only deliver active power to the grid in response to a change in system frequency outside the deadband. The pro- posed algorithm is designed to optimally coordinate a large number of battery assets during the delivery period.

System operators typically re-dispatch outputs of individual battery systems based on forecasts/real-time information of load demand and renewable generation. This could result in an ineffective dispatching because: $i$ ) forecasts may not be accurate enough; ii) real-time measurements might be imperfect. In addition, the efficiency of BESS could vary during their operation. To deal with these issues, the regret minimization framework is adopted. Each battery unit is assumed to be a player and the decision to be made is on the BESS power outputs. Each battery agent is equipped locally with a global information estimator and a power scheduling controller (PSC). The estimator adopts a consensus-based distributed algorithm based on information received by neighbouring agents to estimate the system active power mismatch. A PSC is embedded to execute locally the proposed algorithm and generate an optimal power reference. At the beginning of each time slot $t$, only the last information about renewable generation, load demand and frequency measurement is available to each battery system. Each PSC receives the current frequency measurement, state of charge, and the real-time price set by the system operator at each time $t$. The embedded PSC solves the optimisation problem (4) based on the available information. At the end of the time slot $t$, the actual information is recorded and used by each PSC for dispatching the corresponding battery unit in the next time slot $t+1 \in \mathcal{T}$.

\section{DisTRIBUTED ONLINE OPTIMAL MANAGEMENT OF BESS}

\section{A. OCO Algorithm}

A distributed optimisation algorithm is proposed for (4) using the OCO framework. To facilitate the proposed design, let $h_{t}\left(P_{B, t}\right):=(4 \mathrm{~d})=\sum_{i \in B} P_{B, t}^{i}-\varrho \Delta P_{M, t}=0$. The focus is mainly on providing a solution when the system suddenly suffers active power imbalance. Letting $\lambda_{t} \in \mathbb{R}^{N_{B}}$ be the Lagrange multipliers associated with $h_{t}\left(P_{B, t}\right)$, therefore the Lagrange function of (4) is given by $\mathcal{L}_{t}\left(P_{B, t}, \lambda_{t}\right):=$ $C_{t}\left(P_{B, t}\right)+\lambda_{t}^{T} h_{t}\left(P_{B, t}\right)$, where $P_{B, t} \in \mathcal{F}_{B}$, with $\mathcal{F}_{B}$ being the feasible set of the optimisation problem defined by $(4 b),(4 c)$, and (4e). Well-known methods to solve the centralised OCO are the Arrow-Hurwicz-Uzawa saddle point or primal-dual algorithms [35]. However, they require a central controller to access the system global information. To solve the problem in a distributed manner, a modified OCO algorithm is illustrated below. In a distributed optimisation scenario there is no centralised agent that can access global information and such have the full knowledge of the global constraint $h_{t}\left(P_{B, t}\right)$ at each time step $t$. A consensus framework is therefore embedded into the proposed distributed optimisation algorithm, so that each battery unit locally estimates the global constraint reflecting the change in its nearby environment and therefore sharing and receiving information only with neighbouring battery units. By doing so, the global constraint at each point in time is decomposed into a sum of local estimates. At the time slot $t$, the $i$ th battery agent makes a decision $P_{B, t}^{i}$, which is obtained 
as follows

$P_{B, t}^{i}=$

$\underset{P_{B}^{i} \in \mathcal{F}_{B, i}}{\arg \min } \nabla C_{i, t-1}\left(P_{B, t-1}^{i}\right)\left(P_{B}^{i}-P_{B, t-1}^{i}\right)+\frac{1}{2 \kappa}\left\|P_{B}^{i}-P_{B, t-1}^{i}\right\|$

$+\nabla h_{i, t-1}\left(P_{B, t-1}^{i}\right)\left(P_{B}^{i}-P_{B, t-1}^{i}\right) \sum_{j=1}^{N_{B}} w_{i j} \lambda_{j, t-1}$,

where $\kappa$ is the stepsize, $\nabla(\cdot)$ is the gradient of $(\cdot)$ w.r.t $P_{B}$ at $P_{B}=P_{B, t-1}^{i}, h_{i, t-1}\left(P_{B, t-1}^{i}\right)$ is the local estimation of the global constraint $h_{t-1}\left(P_{B, t-1}\right)$ and $\lambda_{i, t-1}$ is the corresponding Lagrangian muliplier. Let $\Theta=\left[\theta_{1}, \ldots, \theta_{N_{B}}\right]$, with $\mathbf{1}_{N_{B}}^{T} \Theta=1$ and $\Theta \in \mathbb{R}^{N_{B}}$, where $\theta_{i} \neq 0$ if the $i$ th BESS is able to estimate the total supply-demand mismatch, $\theta_{i}=0$ otherwise. Define $s_{i, t-1}=\partial C_{i, t-1}\left(P_{B, t-1}^{i}\right)+$ $\partial h_{i, t-1}\left(P_{B, t-1}^{i}\right) \sum_{j \in \mathcal{B}} w_{i j} \lambda_{j, t-1}$. The distributed solution for the $i$ th battery agent at time step $t$ is calculated as follows

$$
\begin{aligned}
& P_{B, t}^{i}=\mathcal{P}_{\mathcal{F}_{B}}\left(P_{B, t-1}^{i}-\kappa s_{i, t-1}\right), \\
& \lambda_{i, t}=\sum_{j \in \mathcal{B}} w_{i j} \lambda_{j, t-1}+\varepsilon \sum_{i \in \mathcal{B}} w_{i j} y_{j, t-1}, \\
& y_{i, t}=\sum_{j \in \mathcal{B}} w_{i j} y_{j, t-1}+\Delta h_{i, t-1},
\end{aligned}
$$

where $\Delta h_{i, t-1}=\theta_{i}\left(h_{i, t-1}\left(P_{B, t}^{i}\right)-h_{i, t-1}\left(P_{B, t-1}^{i}\right)\right)$; $\varepsilon$ is a positive stepsize and (6a) has a same solution of the minimization (5), as shown in Appendix A. An auxiliary variable is introduced for each battery unit $i=1, \ldots, N$ and for each time step $t$, i.e., $y_{i, t}$ in (6c). This auxiliary variable represents a consensus-based local estimator to track the function sum $\sum_{i=1}^{N_{B}} h_{i, t}\left(P_{B, t}^{i}\right) / N \quad$ [36], which estimates the active power mismatch over $N$ BESS (note that $N$ is the number of available batteries, depending on the current system configuration). The weighted averaging of the iterates from each neighbour of the battery agent $i$, i.e., $\tilde{y}_{i, t-1}$ as described in Step 1 of the OCO algorithm in Algorithm 1, is used to keep local estimates of any global information. Then, equation (6c) provides an update of the local estimator $y_{i, t}$ at time $t, \forall i$.

The proposed framework can be used by aggregators (for example, a Virtual Storage Plant operator) to optimally manage the battery assets and participate into the real-time market/balancing mechanism, or to provide ancillary services to the grid operator. The framework could also be adopted by system operators as a tool to assess the contribution to different ancillary services coming from battery assets available in the whole network and used along with other tools to select in real-time which service providers to use. The co-optimisation frameworks currently adopted by system operators will need to change in order to take into account the different contributions coming from diverse and multiple flexibility service providers and the proposed framework yields a first contribution toward these new tools.

Remark 4.1: The proposed approach could indirectly discern whether a BESS is online and connected. More specifically, when a BESS $i$ is disconnected, this is reflected by a change in the topology of the communication network and thus the corresponding weights $\omega_{i j}$ would change accordingly. As a result, the availability of BESS would be represented by the weighting factors $\omega_{i j}$.

Remark 4.2: The illustrated approach aims at maximising 2the contribution from an arbitrarily large number of BESS to improve the frequency response. Therefore the focus is on BESS and the control action is driven by the amount of active power imbalance that has to be covered by BESS. The BESS power setpoints are continuously updated in a coordinated manner based on the capacity and the current energy stored in each individual storage device, so as to adapt to the timevarying network conditions. The BESS are able to coordinate each other without the need of any central entity and without sharing any local private information with the system operator or the storage aggregator.

The convergence of the proposed OCO algorithm is illustrated in Appendix B.

\section{B. Algorithm Implementation}

Algorithm 1 details the steps of the proposed distributed algorithm.

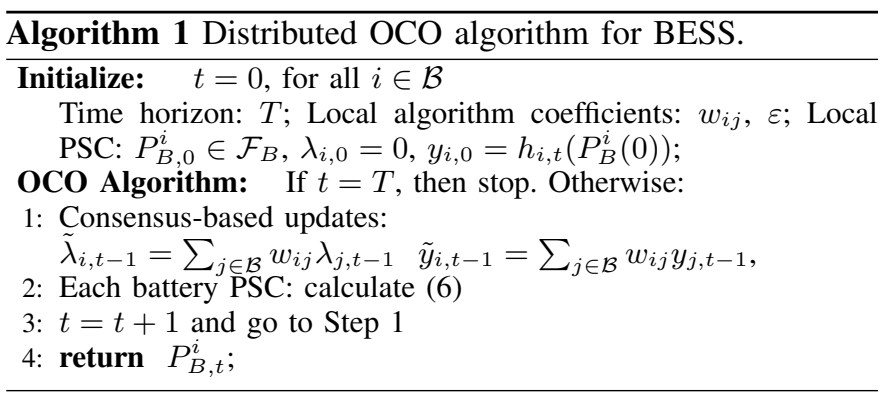

In the proposed scheme, each local PSC is responsible for receiving the generation/demand information of RES and loads from its neighbouring agents through a local communication network, and the reward price broadcast by the system operator in an online fashion. The time horizon of the scheduling process is $T$ and each time slot over the given horizon represents a specific time period, $\Delta t$.

\section{Simulation StUdy}

In this section the distributed solution obtained by (6) is evaluated through two case studies. In Case 5.1 the effectiveness of the proposed distributed OCO algorithm to support frequency regulation is verified for the IEEE 14bus system. The scalability and plug-and-play capability of Algorithm 1 are investigated in Case 5.2, where the IEEE 33bus system is adopted. All systems are modelled using data from MatPower [37]. Results demonstrate that the dispatching BESS can guarantee real-time supply-demand balance with minimal operation costs.

Remark 5.1: The proposed control framework covers the time scales and the control objectives of the traditional primary and secondary control approaches. A comparison with conventional droop-based approaches would not be appropriate since they cannot keep frequency at nominal value, being basically proportional controllers. Although improved and modified droop-based approaches have been proposed in the literature, these fully decentralised approaches are generally less effective since they do not utilise any information exchange and can achieve highly sub-optimal solutions [38]. The 
proposed approach not only targets the same control goals as traditional primary and secondary control, but it also achieves those goals in an optimal and real-time manner, minimising the BESS degradation and power losses, as well as maximising the revenue deriving from the frequency service provision.

\section{A. Simulation setup}

1) Parameters: the system nominal frequency is $f_{0}=50$ Hz. The considered time horizon $\mathcal{T}$ and the reward price is 7.45 £/MW [4]. In order to model an imperfect price signal, a uniform and random noise from $[0,1]$ over $\mathcal{T}$ is added to the price signal. The coefficients $a_{i, t}$ and $b_{i, t}$ are varied and determined by the $\mathrm{SoC}$ and output power at each current point in time [27]. BESS with $13 \mathrm{MWh}$ rated capacity and cell cost of $0.56 \mathrm{f} / \mathrm{W}$ are considered in the optimisation. The SoC operational range is $[20,80] \%$, where each battery has a constant marginal cost. The considered time slot is $\Delta t=0.2 \mathrm{~s}$. The scaling factor $\varrho$ in Problem (4) is set to 1 .

2) Performance metric: to evaluate the performance of the frequency regulation, the frequency deviation from nominal value over a short-time period is calculated as $f_{\mathrm{re}}:=\frac{\bar{f}_{\mathrm{oco}}}{f_{0}} \times$ $100 \%$, where $\bar{f}_{\text {oco }}$ is the mean value of measured system frequency using the proposed OCO algorithm over a given time period. Therefore, $f_{\mathrm{re}} \rightarrow 100 \%$ implies that the regulated frequency gets closer to the nominal value $f_{0}$.

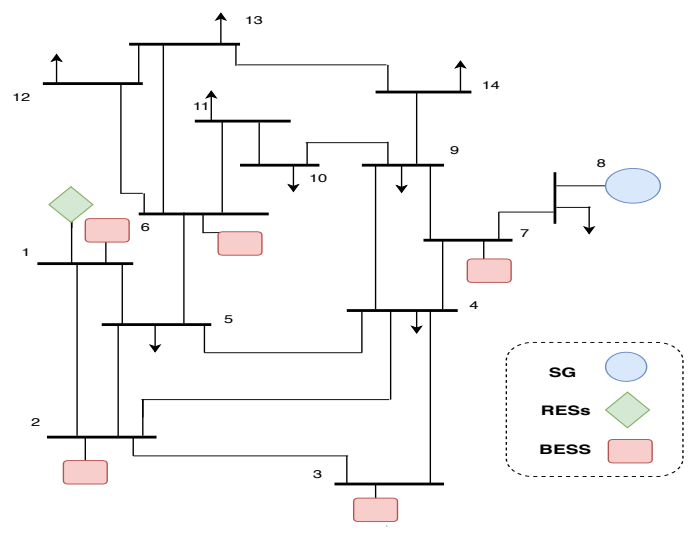

Fig. 1. The single line diagram of IEEE 14-bus with 5 BESS in Case 5.1

\section{B. Case 5.1}

In this case study the IEEE 14-bus with five BESS is considered (see Fig. 1). The BESS maximum power ratings are randomly generated from $[1.2,1.5] \mathrm{MW}$ using a uniform distribution. The inertial constant is $4 \mathrm{~s}$ and the damping is $0.015 \%$. At $t=0$ the system is exposed to an unexpected and sustained supply-demand mismatch, which is modelled as a random variable with a uniform distribution $\mathcal{U}(3.6,4.4) \mathrm{MW}$. BESS are deployed to compensate for this imbalance by using the last available information up to the current point in time. The results are shown in figures 2(a) and 2(c). Fig. 2(a) shows the frequency response with/without the constraints (4e), which limits the BESS ramp-rate proportionally to RoCoF. It can be observed that the proposed framework can quickly restore frequency and the performance of the frequency regulation is improved by introducing the constraints (4e) in (4). The frequency nadir is reduced (see Fig. 2(a)) and the value of the performance indicator $f_{\mathrm{re}}$ over the regulation time (from $5 \mathrm{~s}$ to

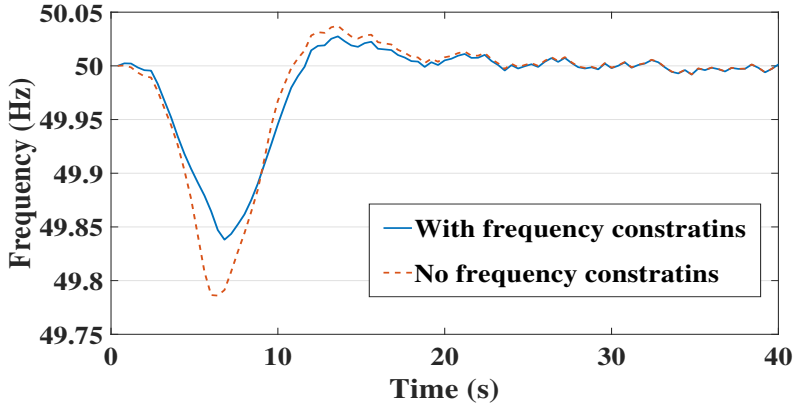

(a) Frequency response

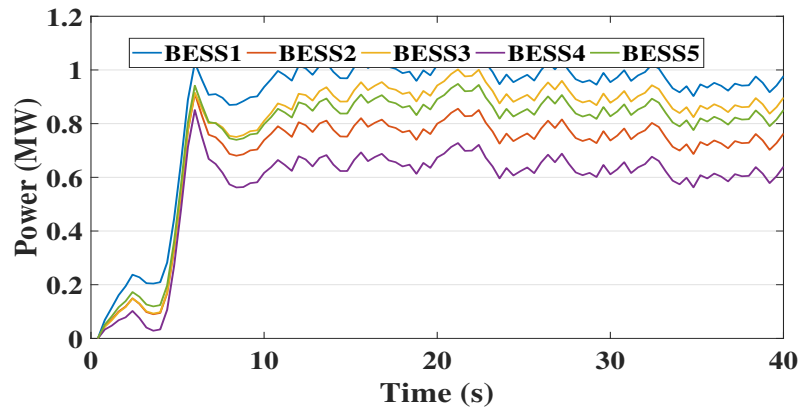

(b) BESS active power outputs

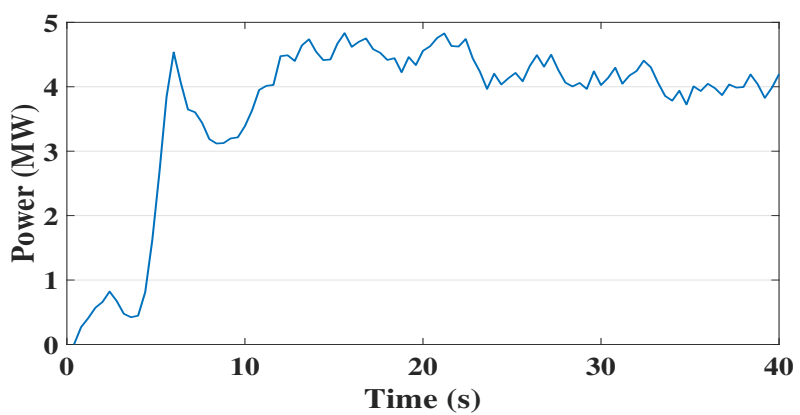

(c) Total active power output of the five BESS

Fig. 2. Results of Case 5.1

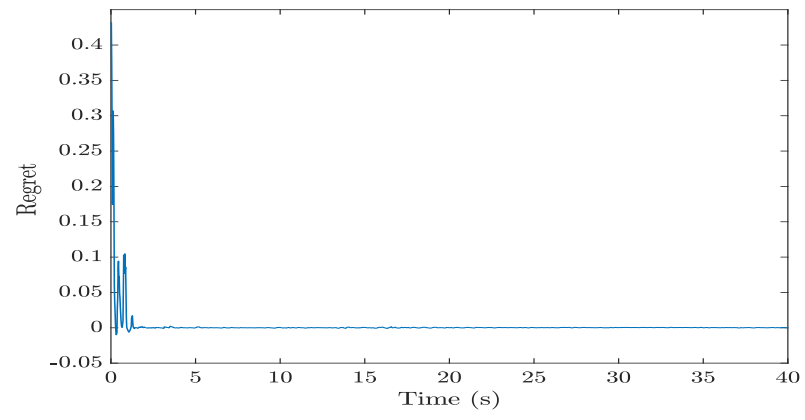

Fig. 3. Evolution of the regret function with time

$15 \mathrm{~s}$ ) is improved from $f_{\mathrm{re}}^{\text {without }}=98.64 \%$ to $f_{\mathrm{re}}^{\text {with }}=99.75 \%$. Fig. 2(b) depicts the BESS output updates. It can be seen that each battery device is re-dispatched within the power ratings. The overall output of the five BESS is given in Fig. 2(c). Furthermore, as shown in Fig. 3, the regret function converges to zero as the time increases, which demonstrates the convergence analysis presented in Appendix B. 


\section{Case 5.2}

In this case study the plug-and-play capability and the scalability of the proposed distributed OCO algorithm is evaluated. Ten BESS with maximum power ratings ranging from 4.8 to 5.5 MW are included into a IEEE 33-bus system. The inertial constant is $6 \mathrm{~s}$ and the damping ratio is $0.015 \%$. At $t=0$ the system is exposed to an unexpected and sustained supplydemand mismatch, which is modelled as a random variable with a uniform distribution $\mathcal{U}(22.5,27.5) \mathrm{MW}$. It is assumed that the connected BESS can still communicate with their neighbours under plug-and-play operations. In this scenario on of BESS, namely BESS7, fails to operate unexpectedly at $t=4 \mathrm{~s}$ during the frequency drop and it is recovered at $t=23 \mathrm{~s}$. Because of its failure BESS7 loses its communication with its neighbouring BESS, and the rest of BESS are re-dispatched accordingly to support the system supply and demand balance. The results are shown in the figures 4(a) - 4(b). Note that the system operator would commonly require the frequency to be restored to the safety range within a pre-defined time, e.g., 30 seconds for the Enhanced Frequency Response. It is shown that the system frequency is regulated to the nominal value within the required time irrespective of the BESS7 failure. After BESS7 is disconnected during the frequency drop, the proposed algorithm is able to timely re-dispatch the outputs of the remaining BESS to new optimal values, in order to continue to regulate the system frequency, as shown in Fig. 4(a). Furthermore, after BESS7 is reconnected, the proposed algorithm re-dispatches the outputs of all BESS again and converges to a new optimal value. This demonstrates that the proposed algorithm can fully manage "leaving and entering" of new battery units and thus support plug-and-play operation without causing significant effect on the frequency response of the system even during the frequency drop.

In order to analyse the algorithm scalability, the computation time taken to calculate the BESS power outputs using the proposed distributed algorithm and an existing centralised method [39] for different number of BESS is calculated and provided in Table II. The results show that, even for 2000 BESS, the proposed distributed algorithm can calculate the power setpoint of each BESS within 0.5s. Differently from the centralised approach, the proposed distributed control approach is suitable for application in large-scale power systems with thousands of BESS.

TABLE II COMPUTATION TIMES

\begin{tabular}{lllllll}
\hline Num. of BESSs & 150 & 300 & 500 & 800 & 1000 & 2000 \\
\hline Time (distributed) & $0.055 \mathrm{~s}$ & $0.0714 \mathrm{~s}$ & $0.128 \mathrm{~s}$ & $0.18 \mathrm{~s}$ & $0.27 \mathrm{~s}$ & $0.49 \mathrm{~s}$ \\
\hline Time (centralised) & $2.17 \mathrm{~s}$ & $3.05 \mathrm{~s}$ & $4.25 \mathrm{~s}$ & $5.41 \mathrm{~s}$ & $6.62 \mathrm{~s}$ & $19.74 \mathrm{~s}$ \\
\hline
\end{tabular}

\section{CONCLUSIONS AND FUTURE WORK}

A distributed control strategy of BESS using a combination of consensus algorithms and online convex optimisation is proposed and illustrated in this paper. To control BESS cooperatively and cost-effectively, a time-varying optimisation problem is formulated while considering uncertain RES generation and demand and BESS degradation costs, and solved in

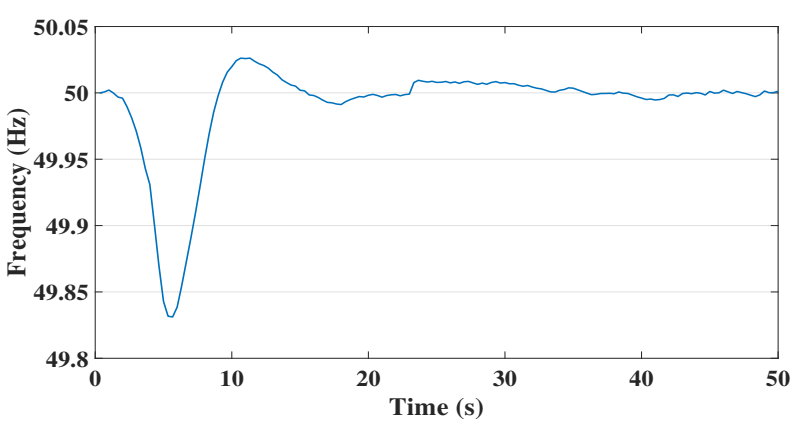

(a) Frequency response

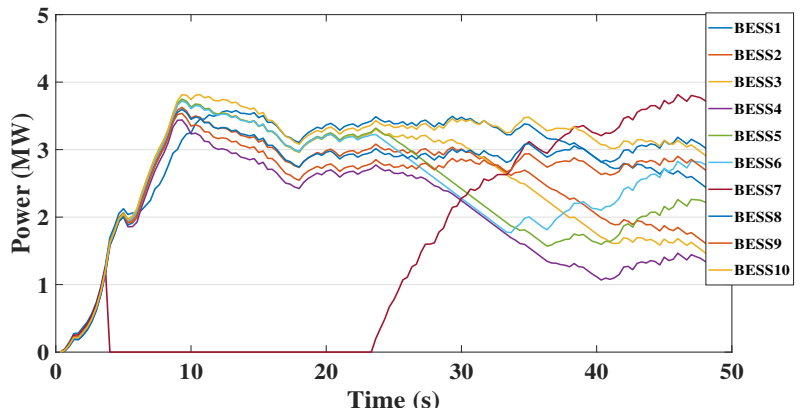

(b) Active power output of the 10 BESS

Fig. 4. Results of Case 5.2

a distributed fashion. The numerical results demonstrate that the system requirements of Enhanced Frequency Response [4] are satisfied by the proposed framework. Furthermore, the proposed approach guarantees the plug-and-play functionality and it is scalable compared with centralised approaches.

Future work includes the incorporation of the BESS location in the network into the control design which is essential when coordinating a large number of BESS to provide system services, as well as the extension of the proposed control framework to include provision of other system services, e.g., voltage regulation and stability and angular stability.

\section{APPENDIX}

\section{A. Proof of the relationship between (5) and (6a)}

We drop the superscript " $i$ " for simplicity.

Proof: (5) can be rewritten as

$$
\begin{aligned}
& P_{B, t} \in \underset{P_{B} \in \mathcal{F}_{B}}{\arg \min } \nabla_{P_{B, t-1}}^{T} L_{t-1}\left(P_{B, t-1}, \lambda_{t-1}\right)\left(P_{B}-P_{B, t-1}\right) \\
& +\frac{1}{2 \kappa}\left\|P_{B}-P_{B, t-1}\right\|^{2}
\end{aligned}
$$

Let $\tilde{P}_{B, t}$ be the minimiser of (5) and $P_{B, t}$ is calculated by (6a). For any $\hat{P}_{B, t} \in \mathcal{F}_{B}$, according to (6a), one has $\left\|P_{B, t}-\left[P_{B, t-1}-\kappa \nabla_{P_{B, t-1}}^{T} L_{t-1}\right]\right\|^{2} \leq$ $\left\|\hat{P}_{B, t}-\left[P_{B, t-1}-\kappa \nabla_{P_{B, t-1}}^{T} L_{t-1}\right]\right\|^{2}, \quad$ where $\nabla_{P_{B, t-1}}^{T} L_{t-1}\left(P_{B, t-1}, \lambda_{t-1}\right)$ is denoted by $\nabla_{P_{B, t-1}}^{T} L_{t-1}$ for simplicity. Note that $\mathcal{F}_{B}$ is a convex set and therefore $P_{B, t}$ is a unique solution of the minimisation 
$\min _{P_{B}} P_{B}-\left[P_{B, t-1}-\alpha \nabla_{P_{B, t-1}}^{T} L_{t-1}\right]$. To show $\tilde{P}_{B, t}=P_{B, t}$, assume that $\tilde{P}_{B, t} \neq P_{B, t}$ and one has

$$
\begin{aligned}
& \left\|P_{B, t}-\left[P_{B, t-1}-\kappa \nabla_{P_{B, t-1}}^{T} L_{t-1}\right]\right\|^{2} \\
& <\left\|\tilde{P}_{B, t}-\left[P_{B, t-1}-\kappa \nabla_{P_{B, t-1}}^{T} L_{t-1}\right]\right\|^{2} .
\end{aligned}
$$

where $\hat{P}_{B, t}$ is replaced by $\tilde{P}_{B, t}$. Calculating the square of both sides of (8), it implies that

$$
\begin{aligned}
& \nabla_{P_{B, t-1}}^{T} L_{t-1}\left(P_{B, t}-P_{B, t-1}\right)+\frac{1}{2 \kappa}\left\|P_{B, t}-P_{B, t-1}\right\|^{2} \\
& <\nabla_{P_{B, t-1}}^{T} L_{t-1}\left(\tilde{P}_{B, t}-P_{B, t-1}\right)+\frac{1}{2 \kappa}\left\|\tilde{P}_{B, t}-P_{B, t-1}\right\|^{2}
\end{aligned}
$$

With the assumption of $\tilde{P}_{B, t} \neq P_{B, t}, \tilde{P}_{B, t}$ should be a minimiser of $\arg \min _{P_{B} \in \mathcal{F}_{B}} \nabla_{P_{B, t-1}}^{T} L_{t-1}\left(P_{B}-P_{B, t-1}\right)+$ $\frac{1}{2 \kappa}\left\|P_{B}-P_{B, t-1}\right\|^{2}$, which implies that for any $\hat{P}_{B, t} \in \mathcal{F}_{B}$,

$$
\begin{aligned}
& \nabla_{P_{B, t-1}}^{T} L_{t-1}\left(\tilde{P}_{B}-P_{B, t-1}\right)+\frac{1}{2 \kappa}\left\|\tilde{P}_{B}-P_{B, t-1}\right\|^{2} \\
& \leq \nabla_{P_{B, t-1}}^{T} L_{t-1}\left(\hat{P}_{B}-P_{B, t-1}\right)+\frac{1}{2 \kappa}\left\|\hat{P}_{B}-P_{B, t-1}\right\|^{2}
\end{aligned}
$$

By letting $\hat{P}_{B, t}=P_{B, t}$, it is obtained that

$$
\begin{aligned}
& \nabla_{P_{B, t-1}}^{T} L_{t-1}\left(\tilde{P}_{B}-P_{B, t-1}\right)+\frac{1}{2 \kappa}\left\|\tilde{P}_{B}-P_{B, t-1}\right\|^{2} \\
& \leq \nabla_{P_{B, t-1}}^{T} L_{p t-1}\left(P_{B}-P_{B, t-1}\right)+\frac{1}{2 \kappa}\left\|_{B}-P_{B, t-1}\right\|^{2},
\end{aligned}
$$

which contradicts the previous results in (8). As a result, it can be concluded that $\tilde{P}_{B, t}=P_{B, t}$, which indicates that (6a) has the same solution as (5).

\section{B. Convergence analysis}

Assumption A.1: The feasible set $\mathcal{F}_{B}$ is compact and the iterates $\left\{\lambda_{i, t}\right\}_{i \in \mathcal{B}, t \geq 1}$ are bounded, i.e., $\left\|P_{B, t}\right\| \leq B_{\mathcal{F}_{B}}$, and $\left\|\lambda_{t}\right\| \leq B_{\lambda}$.

Assumption A.2: $C_{t}(\cdot)$ is bounded on $\mathcal{F}_{B}$, i.e., $\left\|C_{t}(\cdot)\right\| \leq$ $B_{c}$, and is gradient boundedness and Lipschitz continuous with a constant $\mathcal{L}_{c}>0$, i.e. $\left\|\nabla C_{t}(\cdot)\right\| \leq \mathcal{L}_{c}$ and $\left|C_{i, t}(x)-C_{i, t}(y)\right| \leq \mathcal{L}_{c}\|x-y\|, \forall x, y \in \mathcal{F}_{B} . h_{t}(\cdot)$ is bounded on $\mathcal{F}_{B}$, i.e., $\left\|h_{t}(\cdot)\right\| \leq B_{h}$, and has the bounded gradient, i.e., $\left\|\nabla h_{t}(\cdot)\right\| \leq \mathcal{L}_{h}$

Assumption A.3: The sequence $\left\{P_{B, t}^{i}\right\}$ is uniformly bounded, i.e., $U_{p}:=\sup _{t \geq 1} \sup _{P_{B, t}^{i} \in \mathcal{F}_{B}}\left\|P_{B, t}^{i}\right\|^{2}<\infty$. Also, $\mathcal{F}_{B}$ has a bounded radius, i.e., $\left\|P_{B, t}^{i}-P_{B, j}(t)\right\| \leq R_{\mathcal{F}_{B}}$, $\forall P_{B, t}^{i}, P_{B, j}(t) \in \mathcal{F}_{B}$.

Note that Assumptions A.1-A.3 are widely used in the saddlepoint-based OCO framework [22], [36], [39]. The Lemmas introduced in the following are convenient to the proof of Theorem 16.

Lemma A.1: Let $\Delta_{y_{i}}:=\frac{1}{2}\left(\left\|y_{i, t+1}\right\|^{2}-\left\|y_{i, t}\right\|^{2}\right)$ where $\tilde{y}_{i, t}:=\sum_{i j} w_{i j} y_{i, t}$. It follows that $\sum_{i}^{N_{B}} \Delta_{y_{i}} \leq \sum_{i}^{N_{B}} y_{i, t} \nabla$ $\left.h_{i, t}\left(P_{B, t}^{i}\right)\left(P_{B, t+1}^{i}-P_{B, t}^{i}\right)\right)+2 \Theta^{2} R_{\mathcal{F}_{B}}^{2} \mathcal{L}_{h}^{2}$.
Proof:

$$
\begin{aligned}
& \sum_{i}^{N_{B}}\left\|y_{i, t+1}\right\|^{2}=\sum_{i}^{N_{B}}\left\|\tilde{y}_{i, t}+\theta_{i} \nabla h_{i, t}\left(P_{B, t}^{i}\right)\left(P_{B, t+1}^{i}-P_{B, t}^{i}\right)\right\|^{2} \\
& =\sum_{i}^{N_{B}}\left(\left\|\tilde{y}_{i, t}\right\|^{2}+2 \tilde{y}_{i, t} \theta_{i} \nabla h_{i, t}\left(P_{B, t}^{i}\right)\left(P_{B, t+1}^{i}-P_{B, t}^{i}\right)\right. \\
& \left.+\theta_{i}^{2}\left\|\nabla h_{i, t}\left(P_{B, t}^{i}\right)\left(P_{B, t+1}^{i}-P_{B, t}^{i}\right)\right\|^{2}\right) \\
& \leq \sum_{i}^{N_{B}}\left(\left\|y_{i, t}\right\|^{2}+2 y_{i, t} \nabla h_{i, t}\left(P_{B, t}^{i}\right)\left(P_{B, t+1}^{i}-P_{B, t}^{i}\right)\right) \\
& +\Theta^{2} R_{\mathcal{F}_{B}}^{2} \mathcal{L}_{h}^{2}
\end{aligned}
$$

where the first order approximation of $h_{t}\left(P_{B, t+1}\right)$, and $\sum_{i}^{N_{B}} \theta_{i}=1, \sum_{i}^{N_{B}} \tilde{y}_{i, t}=\sum_{i}^{N_{B}} \sum_{j}^{N_{B}} w_{i j} y_{i, t}=\sum_{i}^{N_{B}} y_{i, t}$ are used. The last inequality comes from the bounded gradient and radius. The proof is completed by rearranging the terms in Lemma A.1.

Lemma A.2: Considering (6) and defining $\Delta:=\sum_{i}^{N_{B}} \Delta_{y_{i}}$, the following holds $\forall P_{B}^{i} \in \mathcal{F}_{B, i}, i=\left[1, \ldots, N_{B}\right]$, $\Delta \leq \sum_{i=1}^{N_{B}} \sum_{i=1}^{N_{B}}\left(C_{i, t}\left(P_{B}^{i}\right)-C_{i, t}\left(P_{B, t}^{i}\right)\right)+$ $\sum_{i=1}^{N_{B}} \frac{1}{2 \kappa}\left\|P_{B}^{i}-P_{B, t}^{i}\right\|^{2}-\sum_{i=1}^{N_{B}} \frac{1}{2 \kappa}\left\|P_{B}^{i}-P_{B, t+1}^{i}\right\|^{2}+$ $\kappa \sum_{i=1}^{N_{B}}\left\|\nabla C_{i, t}\left(P_{B, t}^{i}\right)\right\|^{2}+B_{0}+2 \Theta^{2} R_{\mathcal{F}_{B}}^{2} \mathcal{L}_{h}^{2}$, where $B_{0}$ is defined later.

Proof: By using the definition of $\Delta$ and Lemma A.1 it is implied that $\left.\Delta \leq \sum_{i}^{N_{B}} y_{i, t} \nabla h_{i, t}\left(P_{B, t}^{i}\right)\left(P_{B, t+1}^{i}-P_{B, t}^{i}\right)\right)+$ $2 \Theta^{2} R_{\mathcal{F}_{B}}^{2} \mathcal{L}_{h}^{2}$. Recall that $P_{B, t+1}=\left[P_{B, t+1}^{1}, \ldots, P_{B, t+1}^{N_{B}}\right]^{T}$ is the optimal solution of the following optimisation problem, i.e, $P_{B, t+1} \in \arg \min _{P_{B} \in \mathcal{F}_{B}} \sum_{i=1}^{N_{B}} \nabla C_{i, t}\left(P_{B, t}^{i}\right)\left(P_{B}^{i}-P_{B, t}^{i}\right)+$ $\sum_{i=1}^{N_{B}} \frac{1}{2 \kappa}\left\|P_{B}^{i}-P_{B, t}^{i}\right\|^{2}+\sum_{i=1}^{N_{B}} \lambda_{i, t} \nabla h_{i, t}\left(P_{B, t}^{i}\right)\left(P_{B}^{i}-P_{B, t}^{i}\right)$, where $\sum_{i}^{N_{B}} \tilde{\lambda}_{i, t}=\sum_{i}^{N_{B}} \sum_{j}^{N_{B}} w_{i j} \lambda_{i, t}=\sum_{i}^{N_{B}} \lambda_{i, t}$. By adding $\sum_{i=1}^{N_{B}} \nabla \varphi_{i}+\sum_{i=1}^{N_{B}} \frac{1}{2 \kappa}\left\|P_{B, t+1}^{i}-P_{B, t}^{i}\right\|^{2}$, where $\varphi_{i}=$ $C_{i, t}\left(P_{B, t}^{i}\right)\left(P_{B, t+1}^{i}-P_{B, t}^{i}\right)$, to both sides of the previous inequality, it is derived the following

$$
\begin{aligned}
& \Delta+\sum_{i=1}^{N_{B}} \nabla \varphi_{i}+\sum_{i=1}^{N_{B}} \frac{1}{2 \kappa}\left\|P_{B, t+1}^{i}-P_{B, t}^{i}\right\|^{2} \\
& \leq \sum_{i=1}^{N_{B}} \nabla \varphi_{i}+\sum_{i=1}^{N_{B}} \frac{1}{2 \kappa}\left\|P_{B, t+1}^{i}-P_{B, t}^{i}\right\|^{2}+2 \Theta^{2} R_{\mathcal{F}_{B}}^{2} \mathcal{L}_{h}^{2} \\
& \left.+\sum_{i}^{N_{B}} y_{i, t} \nabla h_{i, t}\left(P_{B, t}^{i}\right)\left(P_{B, t+1}^{i}-P_{B, t}^{i}\right)\right) .
\end{aligned}
$$

Since $P_{B, t+1}$ is the minimizer of (5), one has that

$$
\begin{aligned}
& (12) \leq \sum_{i=1}^{N_{B}} \frac{1}{2 \kappa}\left\|P_{B}^{i}-P_{B, t}^{i}\right\|^{2}-\sum_{i=1}^{N_{B}} \frac{1}{2 \kappa}\left\|P_{B}^{i}-P_{B, t+1}^{i}\right\|^{2} \\
& \left.+\sum_{i}^{N_{B}} \lambda_{i, t} \nabla h_{i, t}\left(P_{B, t}^{i}\right)\left(P_{B}^{i}-P_{B, t}^{i}\right)\right)+2 \Theta^{2} R_{\mathcal{F}_{B}}^{2} \mathcal{L}_{h}^{2} \\
& +\sum_{i=1}^{N_{B}} \nabla C_{i, t}\left(P_{B, t}^{i}\right)\left(P_{B}^{i}-P_{B, t}^{i}\right) \\
& +\sum_{i}^{N_{B}}\left(y_{i, t}-\lambda_{i, t}\right) \nabla h_{i, t}\left(P_{B, t}^{i}\right)\left(P_{B}^{i}-P_{B, t}^{i}\right)
\end{aligned}
$$


where, by using Lemma 3 in [36], it can be obtained that $\left\|y_{i, t}\right\| \leq C_{y}$, and it can be concluded that the last term in (13) is bounded by $K \mathcal{L}_{g} R_{\mathcal{F}_{B}}$, where $K=B_{y}-B_{\lambda}$. Note that $\lambda_{i, t}$ can be either $\geq 0$ or $<0$. The case of $\lambda_{i, t} \geq 0$ is discussed first. Therefore,

$$
\begin{aligned}
& \Delta+\sum_{i=1}^{N_{B}} \frac{1}{4 \kappa}\left\|P_{B, t+1}^{i}-P_{B, t}^{i}\right\|^{2} \leq \sum_{i=1}^{N_{B}}\left(C_{i, t}\left(P_{B}^{i}\right)-C_{i, t}\left(P_{B, t}^{i}\right)\right) \\
& +2 \Theta^{2} R_{\mathcal{F}_{B}}^{2} \mathcal{L}_{h}^{2}+B_{0}+\kappa \sum_{i=1}^{N_{B}}\left\|\nabla C_{i, t}\left(P_{B, t}^{i}\right)\right\|^{2} \\
& +\sum_{i=1}^{N_{B}} \frac{1}{2 \kappa}\left\|P_{B}^{i}-P_{B, t}^{i}\right\|^{2}-\sum_{i=1}^{N_{B}} \frac{1}{2 \kappa}\left\|P_{B}^{i}-P_{B, t+1}^{i}\right\|^{2}
\end{aligned}
$$

where the inequality is obtained by using the convexity of $C_{t}\left(P_{B, t}\right) h_{t}\left(P_{B, t}\right)$, and the Cauchy-Schwarz inequality, i.e., $-\sum_{i=1}^{N_{B}} \nabla^{T} C_{i, t}\left(P_{B, t}^{i}\right)\left(P_{B, t+1}^{i}-P_{B, t}^{i}\right) \leq$ $\sum_{i=1}^{N_{B}}\left(\kappa\left\|\nabla C_{i, t}\left(P_{B, t}^{i}\right)\right\|^{2}+\frac{1}{4 \kappa}\left\|P_{B, t+1}^{i}-P_{B, t}^{i}\right\|^{2}\right)$, and $B_{0}:=$ $\max _{t} \max _{P_{B}}\left\|h_{t+1}\left(P_{B}\right)-h_{t}\left(P_{B}\right)\right\|$. Thus the proof is completed by dropping the non-negative term in LHS of (14). The proof of the case of $\lambda_{i, t}<0$ follows similar lines and it is omitted here.

Theorem A.1: Under Assumptions A.1-A.3 the dynamic regret of the proposed distributed OCO algorithm (6), with $y_{i, 0}=\sum_{i \in \mathcal{B}} P_{B}^{i}(0)-\Delta P_{L}(0)$ and $\lambda_{i, 0}=0$, for $i \in \mathcal{B}$, is upper-bounded by

$$
\begin{aligned}
& R_{T}^{d} \leq B_{0} T+2 \Theta^{2} R_{\mathcal{F}_{B}}^{2} \mathcal{L}_{h}^{2} T+\frac{R_{\mathcal{F}_{B}}^{2} T}{\kappa} \\
& +\frac{R_{\mathcal{F}_{B}}}{\kappa} V\left(\left\{P_{B, t}^{*}\right\}_{t=1}^{T}\right)+\kappa \mathcal{L}_{c}^{2} T+\frac{B_{y} T}{2}
\end{aligned}
$$

where the definition of $V\left(\left\{P_{B, t}^{*}\right\}_{t=1}^{T}\right)$ is provided later.

Proof: Let $P_{B, t}^{i, *} \in \mathcal{F}_{B}$ be the minimizer of (5). By replacing $P_{B}^{i}$ by $P_{B, t}^{i, *}$ in Lemma $A .2$, it can be proved that

$$
\begin{aligned}
& \Delta \leq \sum_{i=1}^{N_{B}} \frac{1}{2 \kappa}\left\|P_{B, t}^{i, *}-P_{B, t}^{i}\right\|^{2}+2 \Theta^{2} R_{\mathcal{F}_{B}}^{2} \mathcal{L}_{h}^{2} \\
& +\sum_{i=1}^{N_{B}}\left(C_{i, t}\left(P_{B, t}^{i, *}\right)-C_{i, t}\left(P_{B, t}^{i}\right)\right)-\sum_{i=1}^{N_{B}} \frac{1}{2 \kappa}\left\|P_{B, t}^{i, *}-P_{B, t+1}^{i}\right\|^{2} \\
& +\kappa \sum_{i=1}^{N_{B}}\left\|\nabla C_{i, t}\left(P_{B, t}^{i}\right)\right\|^{2}+B_{0}
\end{aligned}
$$

Note that two distance terms (16) are bounded by $\left\|P_{B, t}^{i, *}-P_{B, t}^{i}\right\|^{2}-\left\|P_{B, t}^{i, *}-P_{B, t+1}^{i}\right\|^{2} \leq$ $2 R_{\mathcal{F}_{B}}\left\|P_{B, t}^{i, *}-P_{B, t-1}^{i, *}\right\|+\left\|P_{B, t}^{i}-P_{B, t-1}^{i, *}\right\|^{2}-$ $\left\|P_{B, t}^{i, *}-P_{B, t+1}^{i}\right\|^{2}$ where the bounded radius of $\mathcal{F}_{B, i}$ in Assumption A.3 is used to obtain the last inequality. Using the bound in (16) and the property of telescoping series, which is a technique to cancel part of each term with part of the next term, and summing up (16) over $t=[1, \ldots, T]$, the following inequality is derived

$$
\begin{aligned}
& \left.\frac{1}{2} \sum_{i=1}^{N_{B}}\left(\left\|y_{i, T+1}\right\|^{2}-\left\|y_{i, 1}\right\|^{2}\right)\right) \\
& \leq \sum_{t=1}^{T} \sum_{i=1}^{N_{B}}\left(C_{i, t}\left(P_{B, t}^{i, *}\right)-C_{i, t}\left(P_{B, t}^{i}\right)\right)+B_{0} T \\
& +\frac{R_{\mathcal{F}_{B}}}{\kappa} V\left(\left\{P_{B, t}^{*}\right\}_{t=1}^{T}\right)+\kappa \mathcal{L}_{c}^{2} T+\frac{R_{\mathcal{F}_{B}}^{2}}{\kappa}+2 \Theta^{2} R_{\mathcal{F}_{B}}^{2} \mathcal{L}_{h}^{2} T,
\end{aligned}
$$

where the last inequality is obtained from the definition of the accumulated variation of the per-slot minimiser, i.e., $V\left(\left\{P_{B, t}^{*}\right\}_{t=1}^{T}\right):=\sum_{t=1}^{T} \sum_{i=1}^{N_{B}}\left\|P_{B, t}^{i, *}-P_{B, t-1}^{i, *}\right\|$, and the bounded radius $R_{\mathcal{F}_{B}}$. Lastly, using the definition of dynamic regret it is deduced that $R_{T}^{d} \leq+B_{0} T+2 \Theta^{2} R_{\mathcal{F}_{B}}^{2} \mathcal{L}_{h}^{2} T+$ $\frac{R_{\mathcal{F}_{B}}^{2} T}{\kappa}+\frac{R_{\mathcal{F}_{B}}}{\kappa} V\left(\left\{P_{B, t}^{*}\right\}_{t=1}^{T}\right)+\kappa \mathcal{L}_{c}^{2} T+\frac{B_{y} T}{2}$, where $\left\|y_{i, T+1}^{2}\right\| \geq$ 0 .

\section{REFERENCES}

[1] R. Doherty, A. Mullane, G. Nolan, D. J. Burke, A. Bryson, and M. O'Malley, "An assessment of the impact of wind generation on system frequency control," IEEE Trans. Power Syst., vol. 25, no. 1, pp. $452-460,2010$.

[2] A. Adrees and J. V. Milanovic, "Study of frequency response in power system with renewable generation and energy storage," in 2016 Power Systems Computation Conference (PSCC), June 2016, pp. 1-7.

[3] A. E. M. Operator, "International review of frequency control adaptation," Tech. Rep., 2016.

[4] Enhanced frequency response. [Online]. Available: https://www. nationalgrideso.com/balancing-services/frequency-response-services

[5] D. Kottick, M. Blau, and D. Edelstein, "Battery energy storage for frequency regulation in an island power system," IEEE Trans. Energy Convers., vol. 8, no. 3, pp. 455-459, 1993.

[6] K. Divya and J. Østergaard, "Battery energy storage technology for power systems-an overview," Electric Power Systems Research, vol. 79, no. 4, pp. 511-520, 2009.

[7] J. Cao, W. Du, and H. Wang, "An improved corrective security constrained opf with distributed energy storage," IEEE Trans. Power Syst., vol. 31, no. 2, pp. 1537-1545, 2016.

[8] Y. Wen, W. Li, G. Huang, and X. Liu, "Frequency dynamics constrained unit commitment with battery energy storage," IEEE Trans. Power Syst., vol. 31, no. 6, pp. 5115-5125, 2016.

[9] S. Pulendran and J. E. Tate, "Energy storage system control for prevention of transient under-frequency load shedding," IEEE Trans. Smart Grid, vol. 8, no. 2, pp. 927-936, 2017.

[10] E. Hsieh and R. Johnson, "Frequency response from autonomous battery energy storage," in Cigré Grid of the Future Symposium, 2012, pp. 1-7.

[11] G. Delille, B. Francois, and G. Malarange, "Dynamic frequency control support by energy storage to reduce the impact of wind and solar generation on isolated power system's inertia," IEEE Trans. Sustainable Enery, vol. 3, no. 4, pp. 931-939, 2012.

[12] I. Egido, L. Sigrist, E. Lobato, L. Rouco, A. Barrado, P. Fontela, and J. Magriñá, "Energy storage systems for frequency stability enhancement in small-isolated power systems," Energy, vol. 13, 2015.

[13] T. Zhao and Z. Ding, "Distributed agent consensus-based optimal resource management for microgrids," IEEE Transactions on Sustainable Energy, vol. 9, no. 1, pp. 443-452, Jan 2018.

[14] Y. Xu, W. Zhang, G. Hug, S. Kar, and Z. Li, "Cooperative control of distributed energy storage systems in a microgrid," IEEE Trans. Smart Grid, vol. 6, no. 1, pp. 238-248, Jan 2015.

[15] A. Kargarian, Y. Fu, and Z. Li, "Distributed security-constrained unit commitment for large-scale power systems," IEEE Trans. Power Syst., vol. 30, no. 4, pp. 1925-1936, 2015.

[16] Y. Xu, J. Hu, W. Gu, W. Su, and W. Liu, "Real-time distributed control of battery energy storage systems for security constrained dc-opf," IEEE Trans. Smart Grid, pp. 1-1, 2016.

[17] T. Zhao and Z. Ding, "Cooperative optimal control of battery energy storage system under wind uncertainties in a microgrid," IEEE Trans. Power Syst., vol. 33, no. 2, pp. 2292-2300, March 2018. 
[18] S. Shalev-Shwartz et al., "Online learning and online convex optimization," Foundations and Trends $囚$ in Machine Learning, vol. 4, no. 2, pp. 107-194, 2012.

[19] W.-J. Ma, V. Gupta, and U. Topcu, "Distributed charging control of electric vehicles using online learning," IEEE Trans. Autom. Control, vol. 62, no. 10, pp. 5289-5295, 2017.

[20] S.-J. Kim and G. B. Giannakis, "An online convex optimization approach to real-time energy pricing for demand response," IEEE Trans. Smart Grid, vol. 8, no. 6, pp. 2784-2793, 2017.

[21] T. Li and M. Dong, "Real-time residential-side joint energy storage management and load scheduling with renewable integration," IEEE Trans. Smart Grid, vol. 9, no. 1, pp. 283-298, Jan 2018.

[22] T. Chen, Q. Ling, and G. B. Giannakis, "An online convex optimization approach to proactive network resource allocation," IEEE Trans. Signal Process., vol. 65, no. 24, pp. 6350-6364, Dec 2017.

[23] Y. Xu, W. Zhang, W. Liu, X. Wang, F. Ferrese, C. Zang, and H. Yu, "Distributed subgradient-based coordination of multiple renewable generators in a microgrid," IEEE Trans. Power Syst., vol. 29, no. 1, pp. 23-33, 2014.

[24] K. I. Tsianos and M. G. Rabbat, "Distributed consensus and optimization under communication delays," in 2011 49th Annual Allerton Conference on Communication, Control, and Computing (Allerton). IEEE, 2011, pp. $974-982$.

[25] C. N. Hadjicostis and T. Charalambous, "Average consensus in the presence of delays in directed graph topologies," IEEE Transactions on Automatic Control, vol. 59, no. 3, pp. 763-768, 2013.

[26] Y. Wen, C. Guo, D. S. Kirschen, and S. Dong, "Enhanced securityconstrained opf with distributed battery energy storage," IEEE Trans. Power Syst., vol. 30, no. 1, pp. 98-108, 2015.

[27] J. Choi, I. Choi, G. An, and D. J. Won, "Advanced power sharing method for energy efficiency improvement of multiple battery energy storages system," IEEE Trans. Smart Grid, 2016.

[28] Y. Shi, B. Xu, D. Wang, and B. Zhang, "Using battery storage for peak shaving and frequency regulation: Joint optimization for superlinear gains," IEEE Trans. Power Syst., vol. 33, no. 3, pp. 2882-2894, 2018.

[29] B. Xu, J. Zhao, T. Zheng, E. Litvinov, and D. S. Kirschen, "Factoring the cycle aging cost of batteries participating in electricity markets," IEEE Trans. Power Syst., vol. 33, no. 2, pp. 2248-2259, March 2018.

[30] D. Greenwood, K. Y. Lim, C. Patsios, P. Lyons, Y. S. Lim, and P. Taylor, "Frequency response services designed for energy storage," Applied Energy, vol. 203, pp. 115-127, 2017.

[31] Ds3 system services volume capped. [Online]. Available: https://http://www.eirgridgroup.com/site-files/library/EirGrid/ Industry-Forum-Slides-Volume-Capped.pdf

[32] A. Ulbig, T. S. Borsche, and G. Andersson, "Impact of low rotational inertia on power system stability and operation," IFAC Proceedings Volumes, vol. 47, no. 3, pp. 7290-7297, 2014.

[33] O. Beltran, R. Peña, J. Segundo, A. Esparza, E. Muljadi, and D. Wenzhong, "Inertia estimation of wind power plants based on the swing equation and phasor measurement units," Applied Sciences, vol. 8 no. 12, p. 2413, 2018.

[34] B. M. Gundogdu, S. Nejad, D. T. Gladwin, M. P. Foster, and D. A. Stone, "A battery energy management strategy for uk enhanced frequency response and triad avoidance," IEEE Trans. Indus. Electron., vol. 65, no. 12 , pp. 9509-9517, 2018

[35] K. Arrow, L. Hurwicz, and H. Uzawa, Studies in Linear and Non-Linear Programming: Stanford Mathematical Studies in the Social Sciences. Literary Licensing, LLC, 2012, no. no. 2. [Online]. Available: https://books.google.co.uk/books?id=ZKtSMQEACAAJ

[36] S. Lee and M. M. Zavlanos, "On the sublinear regret of distributed primal-dual algorithms for online constrained optimization," arXiv preprint arXiv:1705.11128, 2017

[37] R. D. Zimmerman, C. E. Murillo-Sánchez, R. J. Thomas et al., "Matpower: Steady-state operations, planning, and analysis tools for power systems research and education," IEEE Trans. Power Syst., vol. 26, no. 1, pp. 12-19, 2011.

[38] Y. Xu and Z. Li, "Distributed optimal resource management based on the consensus algorithm in a microgrid," IEEE Trans Ind. Electron., vol. 62, no. 4, pp. 2584-2592, April 2015.

[39] A. Koppel, F. Y. Jakubiec, and A. Ribeiro, "A saddle point algorithm for networked online convex optimization," IEEE Trans. Signal Process., vol. 63 , no. 19 , pp. 5149-5164, 2015. 